\title{
Prospective randomized controlled study to compare the postoperative analgesic efficacy of intravenous dexmedetomidine and pethidine on patients undergoing lumbosacral spine surgeries
}

\author{
S Sindhuja \\ Assistant Professor, Dept. of Anaesthesia, KMCT Medical College, Kerala, India \\ *Corresponding Author: S Sindhuja \\ Email: sindhuja.ani@gmail.com
}

Received: $7^{\text {th }}$ May, 2018

Accepted: $13^{\text {th }}$ September, 2018

\begin{abstract}
Introduction: Spine surgeries, especially spine fusion surgeries cause severe postoperative pain, hampering convalescence. This is a randomized double blind controlled study comparing the analgesic efficacy of postoperative intravenous pethidine and dexmedetomidine in patients undergoing lumbosacral spine surgeries.

Materials and Methods: After connecting patients to vital parameters monitor, patients were given general anaesthesia, induced with fentanyl $2 \mathrm{mcg} / \mathrm{kg}$, propofol $2 \mathrm{mg} / \mathrm{kg}$ in graded doses and rocuronium $0.6 \mathrm{mg} / \mathrm{kg}$ to facilitate tracheal intubation. All patients received 1 gram paracetamol iv slow infusion intraoperatively. After one and a half hours of surgery, injection fentanyl $1 \mathrm{mcg} / \mathrm{kg}$ was repeated. After extubation and shifting the patients to PACU, patients were randomly allotted either group P/pethidine group or group D/dexmedetomidine group by computerised randomization. Group P patients received injection pethidine $0.5 \mathrm{mg} / \mathrm{kg}$ loading dose diluted in $5 \mathrm{ml}$ of distilled water slow iv, followed by $0.15 \mathrm{mg} / \mathrm{kg} / \mathrm{hr}$ infusion for $24 \mathrm{hrs}$. Group D patients received injection dexmedetomidine $1 \mathrm{mcg} / \mathrm{kg}$ bolus over 15 minutes followed by infusion of $0.5 \mathrm{mcg} / \mathrm{kg} / \mathrm{min}$. Pulse rate, blood pressure, oxygen saturation, respiratory rate, VAS score and sedation score were monitored every 5 minutes for first 15 minutes, every 15 minutes for 1 hour, every hour till 6th hour, then $12^{\text {th }}$ hour, and $24^{\text {th }}$ hour.

Results: Dexmedetomidine has a faster on set of analgesic effect (10 minutes) compared to pethidine (30 minutes), provides better analgesia and manageable hemodynamics but, causes more sedation with an average sedation score of 2.83 compared to pethidine. Conclusion: Dexmedetomidine is a better analgesic in the postoperative period compared to pethidine.
\end{abstract}

Keywords: Dexmedetomidine, Lumbosacral spine surgeries, Pethidine.

\section{Introduction}

Pain and suffering are an integral part of life. Now pain is recognised as the fifth vital sign. The Taxonomy Committee of International Association for the study of pain defines pain as "An unpleasant sensory and emotional experience associated with actual or potential tissue damage". ${ }^{1}$ Studies show that more than half of patients feel that postoperative pain is their primary concern. ${ }^{2}$ The aims of postoperative pain relief include minimising pain, facilitating recovery, and preventing complications. ${ }^{3}$ Spine surgeries, especially spine fusion surgeries cause severe postoperative pain, hampering convalescence. ${ }^{4}$ Postoperative pain by itself is a risk factor for development of chronic pain syndromes. ${ }^{5,6} \mathrm{We}$ anaesthesiologists have accepted the challenging task of providing pain relief.

Pethidine or meperidine is a synthetic opioid, which acts as an agonist at mu and kappa receptors. It is chosen for our study as part of my institutional practice and also because it produces effective analgesia, equipotent to morphine. It is also advantageous in suppressing postoperative shivering and producing less marked urinary retention and constipation. ${ }^{7}$

Dexmedetomidine, an imidazole compound, is the pharmacologically active dextroisomer of medetomidine that displays specific and selective $\alpha 2$ adrenoceptor agonism. It is chosen for our study because of its several beneficial effects such as excellent analgesia, with lack of respiratory depression and arousable sedation effect that helps in neurological assessment of the patient. It allows psychomotor function to be preserved while letting the patient to rest comfortably. The mechanism of action is unique and differs from clonidine. Post synaptic activation in central nervous system inhibits sympathetic tone and thus decreases blood pressure and heart rate..$^{8-12}$

So in order to find a better post-operative analgesic, we in our study have compared the efficacy of pethidine and dexmedetomidine as post-operative analgesia in patients undergoing lumbosacral spine surgeries between May 2013 and April 2014.

Waleed $\mathrm{M}$ et a ${ }^{15}$ conducted a prospective randomized, double-blinded, controlled study, in which 39 patients with obstructive sleep apnea syndrome undergoing uvulopalatopharyngoplasty were assigned to dexmedetomidine group or Placebo group for postoperative analgesia. Onodera $\mathrm{Y}$ et al ${ }^{16}$ compared the methods used to achieve postoperative analgesia after abdominal aortic surgery with 15 patients each in fentanyl and dexmedetomidine group. Shahbaz R. Arain et $\mathrm{al}^{17}$ conducted a randomized study on 34 patients to check efficacy of dexmedetomidine versus morphine for postoperative analgesia after major inpatient surgery, with 17 in each group. Hence, all the above references are similar to my study, so sample size of 40 each in both groups was taken to remove the bias due to inadequate sample size. 


\section{Materials and Methods}

After obtaining clearance from the Institutional Ethical Committee, 80 patients, both male and female, in the age group of 18 to 70 years, belonging to American Society of Anaesthesiologists (ASA) classes 1 \& 2, undergoing elective lumbosacral spine surgeries at Ganga medical centre, Coimbatore were enrolled in randomized double blind controlled trial. Patients with allergy to the drug being used, ASA 3 patients, ischaemic heart disease, elevated renal parameters and patients who had previously undergone spine surgery were excluded. The study was carried out from May 2013-April 2014. The statistical tests applied were mean, standard deviation and repeated ANOVA Test.

Standard preanesthetic evaluation was performed and informed consent was taken. All the patients were connected to the multiparameter monitors, that measured pulse rate, noninvasive blood pressure, electrocardiography, oxygen saturation and end tidal carbon dioxide. Patients were given general anaesthesia, induced with fentanyl $2 \mathrm{mcg} / \mathrm{kg}$, propofol $2 \mathrm{mg} / \mathrm{kg}$ in graded doses, and rocuronium $0.6 \mathrm{mg} / \mathrm{kg}$ to facilitate tracheal intubation. Injection ramosetron $0.3 \mathrm{mg}$ was also given. After intubation, air entry was checked if it was bilaterally equal, the cuff inflated and tube secured. Air entry rechecked after positioning. Intraoperatively pulse rate, blood pressure, oxygen saturation, respiratory rate and end tidal carbon dioxide were monitored every 5 minutes. The lungs were ventilated to obtain normocapnia that is ETCO2 between 30 to $35 \mathrm{~mm} \mathrm{Hg}$. Maintenance of anaesthesia was with sevoflurane or isoflurane along with nitrous oxide and oxygen. Titrated doses of rocuronium were given to maintain adequate muscle relaxation. All patients received 1 gram iv paracetamol intraoperatively. After one and a half hours of surgery, injection fentanyl $1 \mathrm{mcg} / \mathrm{kg}$ was repeated. All the patients were reversed with injection glycopyrolate $0.01 \mathrm{mg} / \mathrm{kg}$ and neostigmine $0.05 \mathrm{mg} / \mathrm{kg}$ and extubated when fully awake, with sustained eye opening, obeying commands and regularly breathing with normal tidal volume.

All patients were transferred to post anaesthesia care unit and the baseline pulse rate, blood pressure, $\mathrm{SpO} 2$, respiratory rate, visual analogue score for pain and sedation score were noted. All received oxygen 4 $\mathrm{L} /$ minute via face mask for $24 \mathrm{hrs}$. After confirming that the baseline sedation score was less than 3 , the patients were randomly allotted either group P/pethidine group or group $\mathrm{D} /$ dexmedetomidine group by computerised randomization. Group $\mathrm{P}$ patients received injection pethidine $0.5 \mathrm{mg} / \mathrm{kg}$ loading dose diluted in $5 \mathrm{ml}$ of distilled water slow iv, followed by $0.15 \mathrm{mg} / \mathrm{kg} / \mathrm{hr}$ infusion for $24 \mathrm{hrs}$. This dose was chosen because Samina et $\mathrm{al}^{13}$ had given pethidine at the same dose for post operative analgesia in patients undergoing caesarean section. Also, in the study done by K. L. Austin et al, ${ }^{14}$ dose of pethidine was $25 \mathrm{mg} /$ hour. Even at this dose no significant toxic effects of norpethidine were observed and hence there is no risk of norpethidine toxicity in our study. Group D patients received intravenous dexmedetomidine 1 microgram $/ \mathrm{kg}$ loading dose diluted in $20 \mathrm{ml}$ given over 15 minutes via infusion pump followed by $0.5 \mathrm{micrograms} / \mathrm{kg} / \mathrm{hr}$ maintenance. The dose of dexmedetomidine was chosen according to studies done by Shahbaz R. Arain et $\mathrm{al}^{17}$ and Dholakia et al. ${ }^{22}$

Both the patient and the anaesthesiologist in PACU recording the patient data were blinded from the drugs used for pain relief. Pulse rate, blood pressure, oxygen saturation, respiratory rate, VAS score a nd sedation score were monitored every 5 minutes for first 15 minutes, every 15 minutes for 1 hour, every hour till 6 th hour, then $12^{\text {th }}$ hour, and $24^{\text {th }}$ hour. All patients received tablet Pantoprazole $40 \mathrm{mg}$ once daily. Patients who developed hypotension (systolic $\mathrm{BP}<90$ or mean arterial pressure $<60 \mathrm{mmHg}$ ) were treated with fluids and vasopressors (ephedrine $6 \mathrm{mg}$ boluses), severe bradycardia (HR <45) with injection atropine $0.6 \mathrm{mg}$ iv and vomiting with injection ondansetron $4 \mathrm{mg}$. If patient became drowsy (sedation score $>3$ ), infusion dose was reduced by $0.01 \mathrm{mg} / \mathrm{kg} / \mathrm{hr}$ for pethidine and by $0.1 \mathrm{mcg} / \mathrm{kg} / \mathrm{hr}$ for dexmedetomidine every 15 minutes till sedation score became $<$ or $=3$. When VAS was more than or equal to 5 , rescue analgesic of Pethidine IV $10 \mathrm{mg}$ every $10 \mathrm{~min}$ was given in both groups until VAS becomes less than 5. All adverse effects were documented and treated.

\section{Results}

The two groups of patients were similar with respect to age, sex, weight $\&$ ASA status.

Table 1: Comparison of pulse rate (General linear model of repeated ANOVA)

\begin{tabular}{|l|c|c|c|c|c|}
\hline Pulse Rate & Group(N=40 each) & Mean & $\begin{array}{c}\text { Standard } \\
\text { Deviation }\end{array}$ & F value & p Value \\
\hline 0 min & Pethidine & 83.23 & 14.658 & & \\
& Dexmedetomidine & 84.47 & 15.207 & & \\
\hline 5 min & Pethidine & 80.82 & 14.479 & & \\
& Dexmedetomidine & 74.10 & 14.311 & & \\
& Pethidine & 80.85 & 14.651 & & \\
& Dexmedetomidine & 75.02 & 13.968 & & \\
& Pethidine & 79.57 & 15.821 & & \\
& Dexmedetomidine & 71.95 & 12.866 & & \\
\hline
\end{tabular}




\begin{tabular}{|c|c|c|c|c|c|}
\hline $30 \mathrm{~min}$ & Pethidine & 77.80 & 14.941 & \multirow{20}{*}{2836.9} & \multirow{20}{*}{0.000} \\
\hline & Dexmedetomidine & 69.55 & 12.504 & & \\
\hline \multirow[t]{2}{*}{$45 \mathrm{~min}$} & Pethidine & 76.40 & 14.221 & & \\
\hline & Dexmedetomidine & 67.93 & 12.303 & & \\
\hline \multirow[t]{2}{*}{$60 \mathrm{~min}$} & Pethidine & 77.43 & 13.521 & & \\
\hline & Dexmedetomidine & 67.40 & 12.176 & & \\
\hline \multirow[t]{2}{*}{2 hour } & Pethidine & 76.45 & 14.313 & & \\
\hline & Dexmedetomidine & 67.53 & 11.135 & & \\
\hline \multirow[t]{2}{*}{3 hour } & Pethidine & 77.10 & 14.415 & & \\
\hline & Dexmedetomidine & 67.68 & 11.999 & & \\
\hline \multirow[t]{2}{*}{4 hour } & Pethidine & 77.70 & 14.421 & & \\
\hline & Dexmedetomidine & 66.52 & 10.860 & & \\
\hline \multirow[t]{2}{*}{5 hour } & Pethidine & 78.35 & 15.486 & & \\
\hline & Dexmedetomidine & 66.13 & 11.078 & & \\
\hline \multirow{2}{*}{6 hour } & Pethidine & 78.70 & 14.964 & & \\
\hline & Dexmedetomidine & 67.12 & 10.692 & & \\
\hline \multirow[t]{2}{*}{12 hour } & Pethidine & 78.13 & 15.049 & & \\
\hline & Dexmedetomidine & 67.78 & 11.049 & & \\
\hline \multirow[t]{2}{*}{24 hour } & Pethidine & 76.43 & 14.333 & & \\
\hline & Dexmedetomidine & 66.80 & 10.254 & & \\
\hline
\end{tabular}

Table 1 shows that the decrease in pulse rate among the dexmedetomidine group is significant as compared to pethidine group.

Table 2: Comparison of mean noninvasive blood pressure (General Linear model of Repeated ANOVA)

\begin{tabular}{|c|c|c|c|c|c|}
\hline Mean Arterial Pressure & Group $(\mathrm{N}=40$ each $)$ & Mean & $\begin{array}{l}\text { Standard } \\
\text { Deviation }\end{array}$ & F value & p Value \\
\hline $0 \mathrm{~min}$ & $\begin{array}{c}\text { Pethidine } \\
\text { Dexmedetomidine }\end{array}$ & $\begin{array}{c}95.5 \\
95\end{array}$ & $\begin{array}{l}14.9 \\
11.0\end{array}$ & \multirow{14}{*}{6311.1} & \multirow{14}{*}{0.000} \\
\hline $5 \mathrm{~min}$ & $\begin{array}{c}\text { Pethidine } \\
\text { Dexmedetomidine }\end{array}$ & $\begin{array}{l}95.0 \\
89.8\end{array}$ & $\begin{array}{l}13.8 \\
11.9\end{array}$ & & \\
\hline $10 \mathrm{~min}$ & $\begin{array}{c}\text { Pethidine } \\
\text { Dexmedetomidine }\end{array}$ & $\begin{array}{l}95.0 \\
86.1\end{array}$ & $\begin{array}{l}14.2 \\
10.3\end{array}$ & & \\
\hline $15 \mathrm{~min}$ & $\begin{array}{c}\text { Pethidine } \\
\text { Dexmedetomidine }\end{array}$ & $\begin{array}{l}110.8 \\
97.2\end{array}$ & $\begin{array}{l}15 \\
12\end{array}$ & & \\
\hline $30 \mathrm{~min}$ & $\begin{array}{c}\text { Pethidine } \\
\text { Dexmedetomidine }\end{array}$ & $\begin{array}{l}91.8 \\
82.1\end{array}$ & $\begin{array}{l}14.1 \\
11.5\end{array}$ & & \\
\hline $45 \mathrm{~min}$ & $\begin{array}{c}\text { Pethidine } \\
\text { Dexmedetomidine }\end{array}$ & $\begin{array}{l}93.1 \\
80.9\end{array}$ & $\begin{array}{l}13.6 \\
11.8\end{array}$ & & \\
\hline $60 \mathrm{~min}$ & $\begin{array}{c}\text { Pethidine } \\
\text { Dexmedetomidine }\end{array}$ & $\begin{array}{l}92.6 \\
81.1\end{array}$ & $\begin{array}{c}11.8 \\
9.5\end{array}$ & & \\
\hline 2 hour & $\begin{array}{c}\text { Pethidine } \\
\text { Dexmedetomidine }\end{array}$ & $\begin{array}{l}90.2 \\
80.3\end{array}$ & $\begin{array}{l}12.4 \\
9.7\end{array}$ & & \\
\hline 3 hour & $\begin{array}{c}\text { Pethidine } \\
\text { Dexmedetomidine }\end{array}$ & $\begin{array}{l}89.7 \\
78.5\end{array}$ & $\begin{array}{c}11.0 \\
9\end{array}$ & & \\
\hline 4 hour & $\begin{array}{c}\text { Pethidine } \\
\text { Dexmedetomidine }\end{array}$ & $\begin{array}{l}86.4 \\
83.3\end{array}$ & $\begin{array}{c}9.4 \\
12.4\end{array}$ & & \\
\hline 5 hour & $\begin{array}{c}\text { Pethidine } \\
\text { Dexmedetomidine }\end{array}$ & $\begin{array}{l}87.5 \\
82\end{array}$ & $\begin{array}{c}9.4 \\
13.1\end{array}$ & & \\
\hline 6 hour & $\begin{array}{c}\text { Pethidine } \\
\text { Dexmedetomidine }\end{array}$ & $\begin{array}{l}86.9 \\
80.6\end{array}$ & $\begin{array}{c}9.2 \\
12.3\end{array}$ & & \\
\hline 12 hour & $\begin{array}{c}\text { Pethidine } \\
\text { Dexmedetomidine }\end{array}$ & $\begin{array}{l}88.4 \\
80.5\end{array}$ & $\begin{array}{c}10.1 \\
12\end{array}$ & & \\
\hline 24 hour & $\begin{array}{c}\text { Pethidine } \\
\text { Dexmedetomidine }\end{array}$ & $\begin{array}{l}89.5 \\
80.7\end{array}$ & $\begin{array}{l}11.2 \\
12.0\end{array}$ & & \\
\hline
\end{tabular}


Table 2 shows that there was significant decrease in mean arterial pressure in dexmedetomidine group compared to pethidine group.

Table 3: Comparison of respiratory rate (General Linear model of Repeated ANOVA)

\begin{tabular}{|c|c|c|c|c|c|}
\hline Respiratory rate & $\operatorname{Group}(\mathrm{N}=40$ each $)$ & Mean & $\begin{array}{l}\text { Standard } \\
\text { Deviation }\end{array}$ & F value & p Value \\
\hline $0 \mathrm{~min}$ & $\begin{array}{c}\text { Pethidine } \\
\text { Dexmedetomidine }\end{array}$ & $\begin{array}{l}14.87 \\
15.45\end{array}$ & $\begin{array}{l}1.842 \\
1.947\end{array}$ & \multirow{14}{*}{10357.8} & \multirow{14}{*}{0.000} \\
\hline $5 \mathrm{~min}$ & $\begin{array}{c}\text { Pethidine } \\
\text { Dexmedetomidine }\end{array}$ & $\begin{array}{l}15.03 \\
15.38\end{array}$ & $\begin{array}{l}2.069 \\
1.944\end{array}$ & & \\
\hline $10 \mathrm{~min}$ & $\begin{array}{c}\text { Pethidine } \\
\text { Dexmedetomidine }\end{array}$ & $\begin{array}{l}15.27 \\
15.50\end{array}$ & $\begin{array}{l}1.921 \\
1.695\end{array}$ & & \\
\hline $15 \mathrm{~min}$ & $\begin{array}{c}\text { Pethidine } \\
\text { Dexmedetomidine }\end{array}$ & $\begin{array}{l}15.33 \\
15.13\end{array}$ & $\begin{array}{l}1.900 \\
2.015\end{array}$ & & \\
\hline $30 \mathrm{~min}$ & $\begin{array}{c}\text { Pethidine } \\
\text { Dexmedetomidine }\end{array}$ & $\begin{array}{l}15.13 \\
14.80\end{array}$ & $\begin{array}{l}1.911 \\
2.090\end{array}$ & & \\
\hline $45 \mathrm{~min}$ & $\begin{array}{c}\text { Pethidine } \\
\text { Dexmedetomidine }\end{array}$ & $\begin{array}{l}15.37 \\
14.87\end{array}$ & $\begin{array}{l}1.444 \\
1.742\end{array}$ & & \\
\hline $60 \mathrm{~min}$ & $\begin{array}{c}\text { Pethidine } \\
\text { Dexmedetomidine }\end{array}$ & $\begin{array}{l}15.15 \\
14.75\end{array}$ & $\begin{array}{l}1.442 \\
1.794\end{array}$ & & \\
\hline 2 hour & $\begin{array}{c}\text { Pethidine } \\
\text { Dexmedetomidine }\end{array}$ & $\begin{array}{l}15.23 \\
14.73\end{array}$ & $\begin{array}{l}1.405 \\
1.894\end{array}$ & & \\
\hline 3 hour & $\begin{array}{c}\text { Pethidine } \\
\text { Dexmedetomidine }\end{array}$ & $\begin{array}{l}15.23 \\
14.95\end{array}$ & $\begin{array}{l}1.368 \\
2.264\end{array}$ & & \\
\hline 4 hour & $\begin{array}{c}\text { Pethidine } \\
\text { Dexmedetomidine }\end{array}$ & $\begin{array}{l}15.25 \\
14.63\end{array}$ & $\begin{array}{l}1.565 \\
2.047\end{array}$ & & \\
\hline 5 hour & $\begin{array}{c}\text { Pethidine } \\
\text { Dexmedetomidine }\end{array}$ & $\begin{array}{l}15.25 \\
14.90\end{array}$ & $\begin{array}{l}1.613 \\
1.985\end{array}$ & & \\
\hline 6 hour & $\begin{array}{c}\text { Pethidine } \\
\text { Dexmedetomidine }\end{array}$ & $\begin{array}{l}14.75 \\
14.60\end{array}$ & $\begin{array}{l}1.498 \\
1.795\end{array}$ & & \\
\hline 12 hour & $\begin{array}{c}\text { Pethidine } \\
\text { Dexmedetomidine }\end{array}$ & $\begin{array}{l}14.93 \\
14.82\end{array}$ & $\begin{array}{l}1.366 \\
1.583\end{array}$ & & \\
\hline 24 hour & $\begin{array}{c}\text { Pethidine } \\
\text { Dexmedetomidine }\end{array}$ & $\begin{array}{l}14.72 \\
14.60\end{array}$ & $\begin{array}{l}1.240 \\
1.499\end{array}$ & & \\
\hline
\end{tabular}

In Table 3 shows that though there was a mild decrease in respiratory rate in both the groups, the rate remained within normal range of 12 to $16 / \mathrm{min}$.

Table 4: Comparison of VAS (General Linear model of Repeated ANOVA)

\begin{tabular}{|c|c|c|c|c|c|}
\hline VAS score & Group $(\mathrm{N}=40$ each $)$ & Mean & $\begin{array}{l}\text { Standard } \\
\text { Deviation }\end{array}$ & F value & p Value \\
\hline $0 \mathrm{~min}$ & $\begin{array}{c}\text { Pethidine } \\
\text { Dexmedetomidine }\end{array}$ & $\begin{array}{l}7.25 \\
7.00\end{array}$ & $\begin{array}{c}1.032 \\
.934\end{array}$ & \multirow{9}{*}{1575.6} & \multirow{9}{*}{0.000} \\
\hline $5 \mathrm{~min}$ & $\begin{array}{c}\text { Pethidine } \\
\text { Dexmedetomidine }\end{array}$ & $\begin{array}{l}7.15 \\
5.27\end{array}$ & $\begin{array}{l}.834 \\
.933\end{array}$ & & \\
\hline $10 \mathrm{~min}$ & $\begin{array}{c}\text { Pethidine } \\
\text { Dexmedetomidine }\end{array}$ & $\begin{array}{l}6.05 \\
4.23 \\
\end{array}$ & $\begin{array}{l}1.377 \\
1.271 \\
\end{array}$ & & \\
\hline $15 \mathrm{~min}$ & $\begin{array}{c}\text { Pethidine } \\
\text { Dexmedetomidine }\end{array}$ & $\begin{array}{l}5.55 \\
2.97\end{array}$ & $\begin{array}{l}1.339 \\
1.387\end{array}$ & & \\
\hline $30 \mathrm{~min}$ & $\begin{array}{c}\text { Pethidine } \\
\text { Dexmedetomidine }\end{array}$ & $\begin{array}{l}4.85 \\
2.10\end{array}$ & $\begin{array}{l}1.145 \\
1.128\end{array}$ & & \\
\hline $45 \mathrm{~min}$ & $\begin{array}{c}\text { Pethidine } \\
\text { Dexmedetomidine }\end{array}$ & $\begin{array}{l}4.28 \\
1.70\end{array}$ & $\begin{array}{l}.960 \\
.966\end{array}$ & & \\
\hline $60 \mathrm{~min}$ & $\begin{array}{c}\text { Pethidine } \\
\text { Dexmedetomidine }\end{array}$ & $\begin{array}{l}3.43 \\
1.65\end{array}$ & $\begin{array}{c}1.083 \\
.975\end{array}$ & & \\
\hline 2 hour & $\begin{array}{c}\text { Pethidine } \\
\text { Dexmedetomidine }\end{array}$ & $\begin{array}{l}2.90 \\
1.62\end{array}$ & $\begin{array}{l}1.172 \\
1.005\end{array}$ & & \\
\hline 3 hour & Pethidine & 2.85 & 1.312 & & \\
\hline
\end{tabular}




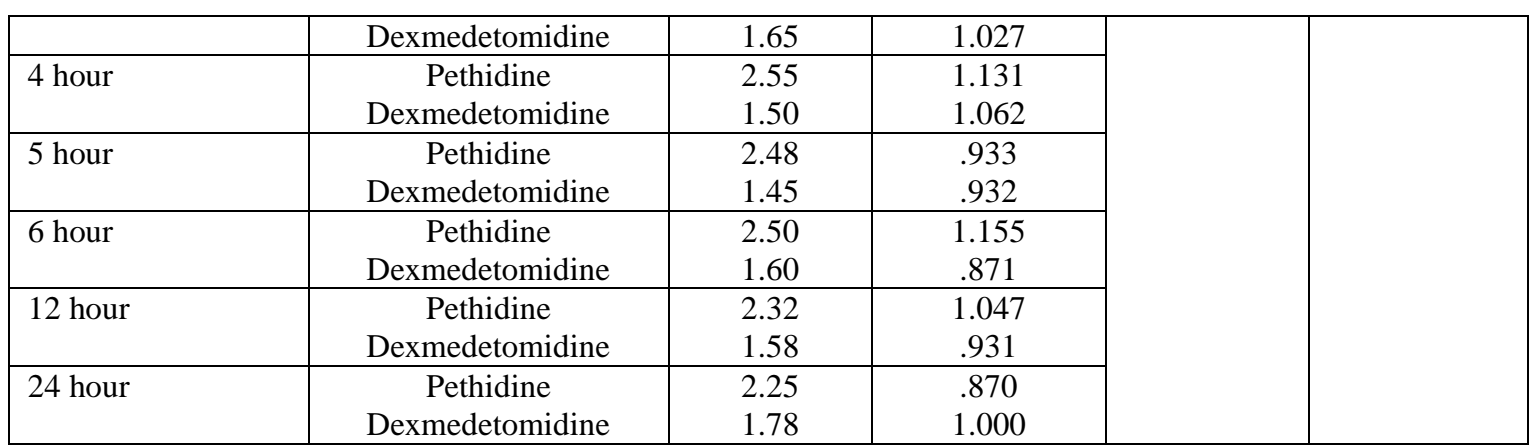

Table 4 shows that dexmedetomidine achieved a lower Visual analogue score compared to pethidine and at a faster rate.

Table 5: Comparison of sedation score (General Linear model of Repeated ANOVA)

\begin{tabular}{|c|c|c|c|c|c|}
\hline Sedation score & Group $(N=40$ each $)$ & Mean & $\begin{array}{l}\text { Standard } \\
\text { Deviation }\end{array}$ & F value & p Value \\
\hline $0 \mathrm{~min}$ & $\begin{array}{c}\text { Pethidine } \\
\text { Dexmedetomidine }\end{array}$ & $\begin{array}{l}1.60 \\
1.68 \\
\end{array}$ & $\begin{array}{l}.496 \\
.474 \\
\end{array}$ & \multirow{14}{*}{5893.2} & \multirow{14}{*}{0.000} \\
\hline $5 \mathrm{~min}$ & $\begin{array}{c}\text { Pethidine } \\
\text { Dexmedetomidine }\end{array}$ & $\begin{array}{l}1.62 \\
1.90\end{array}$ & $\begin{array}{l}.490 \\
.304\end{array}$ & & \\
\hline $10 \mathrm{~min}$ & $\begin{array}{c}\text { Pethidine } \\
\text { Dexmedetomidine }\end{array}$ & $\begin{array}{l}1.80 \\
2.03\end{array}$ & $\begin{array}{l}.405 \\
.276\end{array}$ & & \\
\hline $15 \mathrm{~min}$ & $\begin{array}{c}\text { Pethidine } \\
\text { Dexmedetomidine }\end{array}$ & $\begin{array}{l}1.87 \\
2.48\end{array}$ & $\begin{array}{l}.335 \\
.554\end{array}$ & & \\
\hline $30 \mathrm{~min}$ & $\begin{array}{c}\text { Pethidine } \\
\text { Dexmedetomidine }\end{array}$ & $\begin{array}{l}1.93 \\
2.75\end{array}$ & $\begin{array}{l}.267 \\
.707\end{array}$ & & \\
\hline $45 \mathrm{~min}$ & $\begin{array}{c}\text { Pethidine } \\
\text { Dexmedetomidine }\end{array}$ & $\begin{array}{l}2.03 \\
2.83\end{array}$ & $\begin{array}{l}.158 \\
.636\end{array}$ & & \\
\hline $60 \mathrm{~min}$ & $\begin{array}{c}\text { Pethidine } \\
\text { Dexmedetomidine }\end{array}$ & $\begin{array}{l}2.03 \\
2.70\end{array}$ & $\begin{array}{l}.158 \\
.516\end{array}$ & & \\
\hline 2 hour & $\begin{array}{c}\text { Pethidine } \\
\text { Dexmedetomidine }\end{array}$ & $\begin{array}{l}2.03 \\
2.65\end{array}$ & $\begin{array}{l}.158 \\
.533\end{array}$ & & \\
\hline 3 hour & $\begin{array}{c}\text { Pethidine } \\
\text { Dexmedetomidine }\end{array}$ & $\begin{array}{l}2.03 \\
2.65\end{array}$ & $\begin{array}{l}.158 \\
.533\end{array}$ & & \\
\hline 4 hour & $\begin{array}{c}\text { Pethidine } \\
\text { Dexmedetomidine }\end{array}$ & $\begin{array}{l}2.03 \\
2.70\end{array}$ & $\begin{array}{l}.158 \\
.608\end{array}$ & & \\
\hline 5 hour & $\begin{array}{c}\text { Pethidine } \\
\text { Dexmedetomidine }\end{array}$ & $\begin{array}{l}2.05 \\
2.65\end{array}$ & $\begin{array}{l}.221 \\
.533\end{array}$ & & \\
\hline 6 hour & $\begin{array}{c}\text { Pethidine } \\
\text { Dexmedetomidine }\end{array}$ & $\begin{array}{l}2.07 \\
2.65\end{array}$ & $\begin{array}{l}.267 \\
.483\end{array}$ & & \\
\hline 12 hour & $\begin{array}{c}\text { Pethidine } \\
\text { Dexmedetomidine }\end{array}$ & $\begin{array}{l}2.05 \\
2.55\end{array}$ & $\begin{array}{l}.221 \\
.504\end{array}$ & & \\
\hline 24 hour & $\begin{array}{c}\text { Pethidine } \\
\text { Dexmedetomidine }\end{array}$ & $\begin{array}{l}2.08 \\
2.37\end{array}$ & $\begin{array}{l}.267 \\
.490\end{array}$ & & \\
\hline
\end{tabular}

Table 5 show that the sedation score goes on progressively increasing in the dexmedetomidine group rapidly as compared to the pethidine group.

Table 6: Comparison of requirement of rescue analgesia (by Pearson chi square test)

\begin{tabular}{|c|c|c|c|c|c|}
\hline Group & $\begin{array}{c}\text { No Rescue } \\
\text { Analgesia required }\end{array}$ & $\begin{array}{c}\text { Rescue Analgesia } \\
\text { required }\end{array}$ & Total & 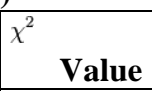 & p Value \\
\hline Pethidine $(\mathrm{N}=40)$ & $25(62.5)$ & $15(37.5)$ & $40(100)$ & \multirow{3}{*}{18.462} & \multirow{3}{*}{0.000} \\
\hline Dexmedetomidine $(\mathrm{N}=40)$ & $40(100)$ & $0(0)$ & $40(100)$ & & \\
\hline Total & $65(81.25)$ & $15(18.75)$ & $80(100)$ & & \\
\hline
\end{tabular}


Table 6 shows that $37.5 \%$ of the pethidine group patients required rescue analgesia while none required rescue analgesia in dexmedetomidine group.

\section{Discussion}

By analysis of variance we found that there was a significant difference in decrease in heart rate and mean arterial pressure in dexmedetomidine group compared to pethidine group. It is interesting to note that even in pethidine group 7 out of $40(17.5 \%)$ patients had MAP> $110 \mathrm{mmHg}$, which can be attributed to no other reason but inadequate pain relief.

Regarding the effect of the drugs on visual analogue scale of patients, there was a $70 \%$ reduction in VAS in pethidine group and $80.8 \%$ in dexmedetomidine group which is statistically significant $(\mathrm{p}<0.001)$. In addition, For the VAS to decrease to a value less than 5 , on an average, it takes 30 minutes in pethidine group whereas, in dexmedetomidine group, it takes 10 minutes, which proves the faster onset of action of dexmedetomidine. Also $37.5 \%$ of the pethidine group required rescue analgesia while not single patient required rescue analgesia in Dexmedetomidine group.

Also, there was a rapid increase in sedation score in the dexmedetomidine group as compared to the pethidine group ie at the end of 45 min the sedation score is 2.03 in pethidine group while it is 2.83 in dexmedetomidine group and $40 \%$ patients requiring a reduction in the infusion dose because of the higher degree of sedation.

There are various studies who have obtained the results similar to the current study. Altindis NT et a $1^{18}$ studied that the addition of dexmedetomidine to pethidine in a patient-controlled analgesia (PCA) device reduced postoperative pethidine consumption when compared to pethidine alone, in 40 patients. Pethidine consumption with PCA was recorded until 24 hours postoperatively. Here, there was a mild insignificant decrease in heart rate and mean arterial pressure. Verbal rating score of pain in the PACU was lower in group I (Dexmedetomidine and pethidine group) than group II (pethidine group $)(\mathrm{p}<0.05)$. Meperidine consumption was lower in group I than group II during the PACU stay and until 24 hours postoperatively $(\mathrm{p}<0.01)$. This is similar to the current study.

The properties of pethidine intravenous infusion in postoperative period was studied by Mitsuhata $\mathrm{H}$ et a ${ }^{19}$ in 2012 who conducted a randomized double-blind controlled study comparing 10 patients receiving continuous intravenous pethidine infusion with 10 patients receiving continuous epidural infusion for postoperative analgesia after upper abdominal surgery.: IV Group $(\mathrm{n}=10)$ received $100 \mathrm{mg} .24 \mathrm{~h}-1$ of pethidine intravenously and saline epidurally, Epi Group $(\mathrm{n}=10)$ received $100 \mathrm{mg} .24 \mathrm{~h}-1$ of pethidine epidurally and saline intravenously. It was found that there were no significant changes in pulse rate, blood pressure, respiratory rate and sedation scales from the baseline in both the groups. This is in accordance with the current study with respect to pethidine characteristics.

Waleed $\mathrm{M}$ et $\mathrm{al}^{15}$ studied analgesic properties of dexmedetomidine infusion after uvulopalatopharyngoplasty in patients with obstructive sleep apnea. The results of the study were: Compared with placebo group, patients in the dexmedetomidine group required $52.7 \%$ less PCA morphine during the first 24 hours postoperatively, with significantly better visual analogue scale scores, less incidence of respiratory obstruction (5 vs. 12 patients, respectively; $\mathrm{P}=.037$ ) and longer time to first analgesic request (21 (11) vs. 9 (4) minutes; $\mathrm{P}=.002$ ) and higher sedation scores and lesser nausea and vomiting(7 vs. 24 patients, respectively; $\mathrm{P}<$ 0.05).

Shahbaz R. Arain et $\mathrm{al}^{17}$ conducted a randomized study on 34 patients undergoing elective inpatient who received either dexmedetomidine (initial loading dose of 1 microgram $/ \mathrm{kg}$ over $10 \mathrm{~min}$ followed by 0.4 microgram $/ \mathrm{kg}(-1) \mathrm{h}(-1)$ for $4 \mathrm{~h}$ ) or morphine sulfate $(0.08 \mathrm{mg} / \mathrm{kg}) 30 \mathrm{~min}$ before the end of surgery up to 24 $\mathrm{h}$. They too found that there was a decrease in heart rate, mean arterial pressure in dexmedetomidine group as compared to morphine group and required less morphine(PACU dexmedetomidine group, 4.5 +/- 6.8 $\mathrm{mg}$; morphine group, $9.2+/-5.2 \mathrm{mg}$ ). Gurbet et $\mathrm{al}^{20} \mathrm{did}$ a randomized 40 patients undergoing total abdominal hysterectomy to receive either dexmedetomidine i.e. study group -loading $1 \mu \mathrm{g} / \mathrm{kg}$ over 30 minutes followed by infusion at $0.5 \mu \mathrm{g} / \mathrm{kg} / \mathrm{h}$ until the end of surgery (same dose as the current study) or $0.9 \%$ saline i.e. control group (with the same infusion protocol) prior to the induction of anaesthesia. Here too patients reported better analgesia and sedation in dexmedetomidine group

Feld et $\mathrm{al}^{21}$ conducted a pilot study among morbidly obese patients undergoing open gastric bypass surgery on post-operative analgesia while morphine was given as rescue analgesia. One group patients received 0.5 $\mathrm{mcg} / \mathrm{kg}$ fentanyl on induction followed by fentanyl infusion $(0.5 \mathrm{mcg} / \mathrm{kg} / \mathrm{h})$ until the end of surgery; and the other received dexmedetomidine (loading dose of 0.5 $\mathrm{mcg} / \mathrm{kg}$ over 10 minutes prior to induction, followed by infusion at $0.4 \mathrm{mcg} / \mathrm{kg} / \mathrm{h}$ ). Dholakia et $\mathrm{al}^{22}$ conducted a retrospective study of patients undergoing laparoscopic bariatric surgery that compared a dexmedetomidine group (loading dose of $1 \mathrm{mcg} / \mathrm{kg}$ over 10 minutes followed by infusion at $0.2-0.7 \mathrm{mcg} / \mathrm{kg} / \mathrm{h}$ until the end of surgery) with control group. The results of both studies were similar to our study.

The sedative properties of dexmedetomidine without affecting respiration was studied and proven by Martin $\mathrm{E}$ et $\mathrm{al}^{23}$ who did double-blind, randomized, placebo-controlled, multicenter trial evaluating Dexmedetomidine for the sedation of 401 post surgical patients in an ICU. Dexmedetomidine was shown to have no effect on the respiratory rate, oxygen desaturation, duration of weaning, or time to extubation. 
All the above detailed study references, that is, Altindis NT et al, ${ }^{18}$ Ekemen et al, Mitsuhata $\mathrm{H}$ et al, ${ }^{19}$ Waleed $\mathrm{M}$ et al, ${ }^{20}$ Shahbaz R. Arain et al, ${ }^{17}$ Gurbet et al, ${ }^{20}$ Feld et $a{ }^{21}{ }^{21}$ Dholakia et $\mathrm{al}^{22}$ and Martin $\mathrm{E}$ et $\mathrm{al}^{23}$ show that their results were in accordance with current study. The dexmedetomidine group produced a decrease in heart rate and mean arterial pressure without significant decrease in respiratory rate with better analgesic property and sedative action as compared pethidine.

\section{Conclusion}

Dexmeditomidine is a better analgesic in the postoperative period compared to pethidine with a faster onset of analgesic effect compared to pethidine. Dexmedetomidine is a potent, highly selective and specific $\alpha_{2}$-adrenoreceptor agonist that has good analgesic property with manageable hemodynamic alterations and more sedation.

\section{Source of Funding: Nil}

\section{Conflict of Interest: Nil}

\section{References}

1. Pain terms: a list with definitions and notes on usage. Recommended by the IASP Subcommittee on Taxonomy Pain. 1979;6(3):249.

2. International Association for the Study of Pain (IASP). Management of acute pain: a practical guideline. Seattle: International Association for the Study of Pain (IASP), 1992.

3. Warfield CA, Kahn CH. Acute pain management. Programs in U.S. hospitals and experiences and attitudes among U.S. adults. Anesthesiol. 1995;83:1090-1094.

4. Sang Hoon Lee, Kyung Hyun Kim, Seong-Mee Cheong, Sumi Kim, Mirang Kooh, Dong Kyu Chin, A Comparison of the Effect of Epidural Patient-Controlled Analgesia with Intravenous Patient-Controlled Analgesia on Pain Control after Posterior Lumbar Instrumented Fusion. J Korean Neurosurg Soc. 2011;50:205-208.

5. Gottschalk A, Freitag M, Tank S, Burmeister MA, Kreil S, Kothe R, Hansen-Algenstedt N, Weisner L, Staude HJ, Standl T Quality of postoperative pain using an intraoperatively placed epidural catheter after major lumbar spinal surgery. J Anesthesiol. 2004;101 (1):175180.

6. Reza Movasghi, Firooz Salehpour, Ali Ahmadvand, Davood Agha, Mohammadi, Abbas Ali Dorosty, Reza Vafaee. The effect of epidural morphine spongein post operative pain control after microdiscectomy. $J$ Paramedical Sci., 2012;3(4):7-11.

7. 'Essentials of medical pharmacology' by K.D Tripathi, chapter 34,page 458-459.

8. Virtanen R, Savola JM, Saano V, Nyman L. Characterization of the selectivity, specificity and potency of dexmedetomidine as an alpha 2-adrenoceptor agonist. Eur J Pharmacol. 1988;150:9-14.

9. Ahlquist RP. A study of the adrenotropic receptors. Am J Physio. 1948;153:586-600.

10. Langer SZ. Presynaptic regulation of catecholamine release. Biochem Pharmacol. 1974;23:1793-1800.

11. Drew GM, Whiting SB. Evidence for two distinct types of postsynaptic alpha-adrenoceptor in vascular smooth muscle in vivo. Br J Pharmacol. 1979;67:207-215.
12. Berthelsen S, Pettinger WA. A functional basis for classification of alpha-adrenergic receptors. Life Sci. 1977;21:595-606.

13. Samina Ismail, Gauhar Afshan, Abdul Monem and Aliya Ahmed. Postoperative analgesia for caesarean section: comparison of patient controlled analgesia with continuous infusion using pethidine. Br J Anaesth. 2012 Sept, paper no 98.

14. K. L. Austin, J. V. Stapleton and L. E. Mather. Rate of Formation of Norpethidine from Pethidine, Br J Anaesth. 1981;53:255.

15. Waleed M. Abdelmageed, Kaled M. Elquesny, Ramadn I. Shabana, Hossam M. Abushama, and Ahmad M. Nassar.Analgesic properties of a dexmedetomidine infusion after uvulopalatopharyngoplasty in patients with obstructive sleep apnea. Saudi J Anaesth. 2011;5(2):150156.

16. Onodera Y, Yamaqishi, Kurosawa A, Takahata O, Iwasaki H. Postoperative analgesia of continuous intravenous fentanyl or dexmedetomedine for patients receiving anticoagulant therapy. Masui. 2011:60:936-940.

17. Arain SR, Ruehlow RM, Uhrich TD, Ebert TJ. The efficacy of dexmedetomidine versus morphine for postoperative analgesia after major inpatient surgery. Anesth Analg. 2004;98(1):153-158.

18. Altindis NT, Karaaslan D, Peker TT, Ozmen S, Bulbul M. Comparison of meperidine alone with meperidine plus dexmedetomidine for postoperative patient-controlled analgesia. Neurosci (Riyadh). 2008;13:117-122.

19. Mitsuhata H, Matsumoto S, Hasegawa J, Ohtaka K. Continuous intravenous pethidine infusion for analgesia after upper abdominal surgery: a randomized, prospective double-blind comparison with continuous epidural infusion of pethidine. 1991;40(12):1770-1776.

20. Gurbet A, Basagan-Mogol E, Turker G, Ugun F, Kaya FN, Ozcan B. Intraoperative infusion of Dexmedetomidine reduces perioperative analgesic requirements. Can J Anaesth. 2006;53:646-652.

21. Feld JM, Hoffman WE, Stechert MM, Hoffman IW, Ananda RC. Fentanyl or Dexmedetomidine combined with Desflurane for bariatric surgery. J Clin Anaesth. 2006;18(1)24-28.

22. Dholakia C, Beverstein G, Garren M, Nemergut C, Boncyk J, Gould JC. The impact of perioperative dexmedetomidine infusion on postoperative narcotic use and duration of stay after laparoscopic bariatric surgery. $J$ Gastrointest Surg. 2007(11):1556-1559.

23. Martin E, Ramsay G, Mantz J, Sum-Ping S T. The Role of the alpha-2 adrenergic Agonist Dexmedetomidine in Postsurgical Sedation in the Intensive Care Unit. $J$ Intensiv Care Med. 2003;18:29-41.

How to cite this article: Sindhuja S. Prospective randomized controlled study to compare the postoperative analgesic efficacy of intravenous dexmedetomidine and pethidine on patients undergoing lumbosacral spine surgeries. Indian $\mathrm{J}$ Clin Anaesth. 2018;5(4):596-602. 